\title{
Eosinophils are required to suppress Th2 responses in Peyer's patches during intestinal infection by nematodes
}

\author{
J Strandmark ${ }^{1}$, S Steinfelder ${ }^{1}, \mathrm{C} \mathrm{Berek}^{2}, \mathrm{AA} \mathrm{Kühl}^{3}, \mathrm{~S}$ Rausch $^{1,4}$ and S Hartmann ${ }^{1,4}$
}

Infections with enteric nematodes result in systemic type 2 helper T (Th2) responses, expansion of immunoglobulin (Ig)G1 antibodies, and eosinophilia. Eosinophils have a supportive role in mucosal Th2 induction during airway hyperreactivity. Whether eosinophils affect the local T-cell and antibody response in the gut-associated lymphoid tissue during enteric infections is unknown. We infected eosinophil-deficient $\triangle$ dbIGATA-1 mice with the Th2-inducing small intestinal nematode Heligmosomoides polygyrus and found that parasite fecundity was decreased in the absence of eosinophils. A lack of eosinophils resulted in significantly augmented expression of GATA-3 and IL-4 by CD4 ${ }^{+}$T cells during acute infection, a finding strictly limited to Peyer's patches (PP). The increase in IL-4-producing cells in $\Delta$ dbIGATA-1 mice was particularly evident within the CXCR5 ${ }^{+} \mathrm{PD}-1^{+}$T-follicular helper cell population and was associated with a switch of germinal centre B cells to IgG1 production and elevated serum IgG1 levels. In contrast, infected wild-type mice had a modest IgG1 response in the PP, whereas successfully maintaining a population of $\lg \mathrm{A}^{+}$ germinal center $B$ cells. Our results suggest a novel role for eosinophils during intestinal infection whereby they restrict IL-4 responses by follicular T helper cells and IgG1 class switching in the PP to ensure maintenance of local IgA production.

\section{INTRODUCTION}

The traditional view of eosinophils as effector cells that evolved to fight parasitic worms originates from the observation that during helminth infections their numbers increase markedly in response to IL- 5 produced by $\mathrm{CD} 4^{+}$type 2 helper T (Th2) cells. ${ }^{1}$ However, for many helminth infections, convincing evidence that they are an indispensible part of the host's immune defence is lacking. ${ }^{2}$ Notwithstanding their ability to contribute to protection against the larval stages of some helminth species, ${ }^{3,4}$ their role is often redundant, ${ }^{5}$ their ablation of no consequence ${ }^{6}$ or their presence even of apparent benefit to the parasite.

Beyond helminth infections, eosinophilia develops in Th2-driven immune disorders, most notably asthma, ${ }^{8}$ but also others such as eosinophilic gastritis, dermatitis, and esophagitis, in all of which they have been ascribed a role linked to immunopathology. ${ }^{9}$

However, eosinophils are also a prominent part of the healthy intestine. ${ }^{10}$ As their granules not only contain cationic proteins with anti-microbial activity, but also an impressive range of pre-formed cytokines and chemokines that can rapidly be released upon appropriate stimulation, eosinophils have great potential to influence homeostatic processes as well as early immunological events occurring upon tissue invasion by pathogens. Accordingly, a more diverse picture of eosinophils has recently emerged in which they function in a number of settings, including bacterial and viral clearance, ${ }^{11,12}$ metabolic homeostasis, ${ }^{13}$ and muscle regeneration. ${ }^{14}$

Recent findings additionally reveal that eosinophils are of major importance for plasma cell survival and maintenance in

${ }^{1}$ Institute of Immunology, Department of Veterinary Medicine, Freie Universität Berlin, Berlin, Germany. ${ }^{2}$ Deutsches Rheuma Forschungszentrum Berlin, a Leibniz Institute, Berlin, Germany and ${ }^{3}$ Department of Internal Medicine, Rheumatology and Clinical Immunology/Research Center ImmunoSciences (RCIS), Charité-University Medicine Berlin, Berlin, Germany. Correspondence: S Rausch (sebastian.rausch@fu-berlin.de)

${ }^{4}$ These authors contributed equally to this work. 
bone marrow ${ }^{15}$ and intestine, ${ }^{16}$ in the latter of which they contribute to the generation of IgA plasma cells in naive mice, thus demonstrating an important role for eosinophils in gut homeostasis.

Whether eosinophils affect antibody responses against intruding pathogens has not been investigated. Here we show for the first time that the lack of eosinophils during infection with an enteric nematode leads to deregulated adaptive local immune responses in the Peyer's patches (PP). Eosinophildeficient mice infected with the natural murine parasite Heligmosomoides polygyrus displayed an increased Th2 response accompanied by sharply elevated immunoglobulin (Ig)G1 class switching by germinal centre B cells in the PP, leading to increased serum levels of IgG1. The increase in Th2 and associated antibody responses as well as a defect in the small intestinal mucous shield coincided with a significant reduction in parasite fecundity.

\section{RESULTS}

\section{H. polygyrus infection results in local and systemic eosinophilia}

To gain insight into the distribution of eosinophils in mice infected with enteric nematodes we compared histological sections of the gut of naive and $H$. polygyrus-infected BALB/C mice at 2 weeks post infection. Eosinophils were readily detectable in the lamina propria of the proximal small intestine and were found in higher numbers after infection (Figure 1a). Furthermore, eosinophils had infiltrated the Peyer's patches (PP) and mesenteric lymph nodes (mLN) (Figure 1a). To assess the kinetics of eosinophil accumulation in more detail we infected wild-type (WT) BALB/c and $\Delta$ dblGATA-1 mice, deficient in eosinophils owing to a deletion of a high-affinity GATA-binding site in the GATA-1 promoter, ${ }^{17}$ and detected eosinophils by flow cytometry according to the gating strategy shown in Figure 1b. The frequency and absolute number of $\mathrm{SSC}^{\text {hi }} \mathrm{CD} 45^{+}$SiglecF ${ }^{+} \mathrm{CCR}^{+}$cells with morphological features of eosinophils (Figure 1c) increased in infected BALB/ $\mathrm{c}$ mice and reached levels significantly higher than those seen in naive controls at 2 weeks post infection in the small intestine, $\mathrm{PP}$, and $\mathrm{mLN}$ (Figure 1d and e). Frequencies of eosinophils also increased in spleen, bone marrow, and peritoneum (Supplementary Figure S1 online). As expected, $\Delta$ dblGATA-1 mice were devoid of eosinophils.

We further detected SSChi ${ }^{\text {FceRI }}{ }^{+}$cells in the lamina propria, which were slightly elevated in $\Delta$ dblGATA-1 mice infected with $H$. polygyrus (Supplementary Figure S1B). These cells appeared to consist mostly of mast cells as indicated by c-kit expression (not shown).

In summary, eosinophils rapidly increase locally and systemically in response to nematode infection in WT mice.

\section{Eosinophils restrict nematode-driven Th2 induction in PP of WT mice}

Eosinophils have been shown to support the induction and tissue accumulation of Th2 cells during airway hyperreactivity. ${ }^{18}$ We therefore asked whether the development of Th2 responses to an intestinal nematode infection was affected in mice lacking eosinophils and analyzed the T-cell response in WT and $\Delta$ dblGATA-1 mice acutely infected with $H$. polygyrus.

Strikingly, as early as 6 days after infection, PP of $\Delta$ dblGATA-1 mice infected with $H$. polygyrus showed significantly elevated frequencies of $\mathrm{CD} 4^{+}$T cells expressing GATA3 , the transcriptional regulator of Th2 cells, compared with infected WT controls (Figure 2a). The stronger Th2 development was restricted to the PP, as frequencies of GATA- $3^{+}$Th2 cells increased to a similar extent in $\mathrm{mLN}$, spleen and the small intestine of infected $\mathrm{WT}$, and $\Delta \mathrm{dblGATA}-1$ mice (Figure $\mathbf{2 b}$ and Supplementary Figure S2A). In accordance with the increased GATA-3 expression, we found that the frequency of $\mathrm{CD}^{+} \mathrm{T}$ cells expressing IL-4 following PMA/ionomycin stimulation was significantly higher in the PP of infected $\Delta$ dblGATA-1 mice compared with infected WT controls at 2 weeks post infection (Figure 2c), whereas IL-4-producing cells increased comparably in the mLN of both strains in response to infection (Figure 2d). We next assessed whether the production of other Th2 associated cytokines was affected by eosinophil deficiency by stimulating cells from the PP and $\mathrm{mLN}$ with antiCD3/28 antibodies and measuring secretion of IL-13, IL-5, and IL-10 in addition to IL-4. In agreement with flow cytometric data, we measured higher levels of IL- 4 secretion by PP cells of infected $\Delta$ dblGATA-1 mice, although the difference did not reach statistical significance (Figure 2e). IL-4 secretion from the mLN of $\triangle$ dblGATA- 1 and BALB/c mice was comparable, as was secretion of all other cytokines by cells from PP and mLN (Figure 2e-h). Furthermore, production of IL-1 $\beta$ and IL-6, both implicated in eosinophil-mediated support of PP B-cell responses $^{15,19}$ and in limiting Th2 induction, ${ }^{20,21}$ was normal in the small intestine of $\triangle$ dblGATA-1 mice infected with H. polygyrus (Figure 2i and $\mathbf{j}$ ).

Given the importance of dendritic cells (DCs) in the instruction of Th cell responses, we assessed the composition of the CD11 $\mathrm{ch}^{\mathrm{hi}} \mathrm{MHCII}^{+}$DC population in the PP and mLN of $\mathrm{BALB} / \mathrm{c}$ and $\triangle \mathrm{dblGATA}-1$ mice and found that $\mathrm{CD} 103^{+} \mathrm{DC}$, previously shown to constitutively produce IL-12 and thereby suppress helminth-induced Th2 responses, ${ }^{22}$ were present at lower levels in the PP of $H$. polygyrus infected $\Delta$ dblGATA-1 mice compared with BALB/c mice (Figure $2 \mathbf{k}$ and $\mathbf{i}$ ).

We found that PP, but not mLN of $\triangle$ dblGATA-1 mice, were smaller than the PP of WT controls (Supplementary Figure S2B). The cellularity of PP corresponded well to previously published studies ${ }^{16,19}$ with PP cell counts of $\triangle$ dblGATA-1 mice being reduced by $\sim 50 \%$ compared with $\mathrm{BALB} / \mathrm{c}$ mice. Frequencies of $\mathrm{CD}^{+} \mathrm{T}$ cells were comparable between WT and $\triangle$ dblGATA-1 mice, but decreased in the mLN in response to $H$. polygyrus infection (Supplementary Figure S2C). This decrease was compensated for by $\mathrm{B}_{220^{+}} \mathrm{B}$ cells, demonstrating that B-cell strongly infiltrate and/or expand in the $\mathrm{mLN}$ in response to H. polygyrus infection (Supplementary Figure S2D). Finally, the frequencies of Foxp ${ }^{+}$regulatory $T$ cells were not affected by the absence of eosinophils (Supplementary Figure S2E). To determine whether the increased expression of GATA-3 in eosinophil-deficient animals was 

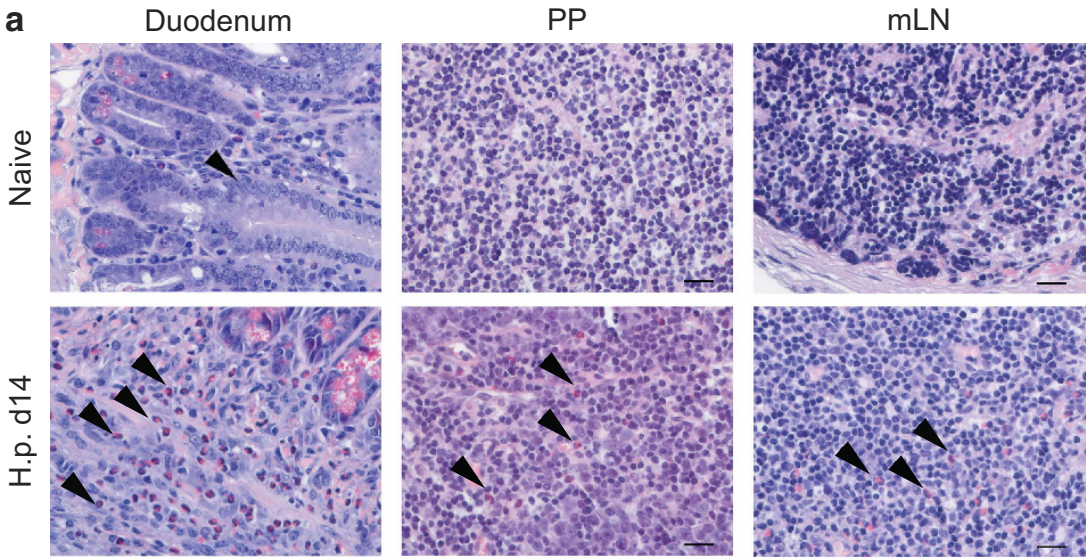

b

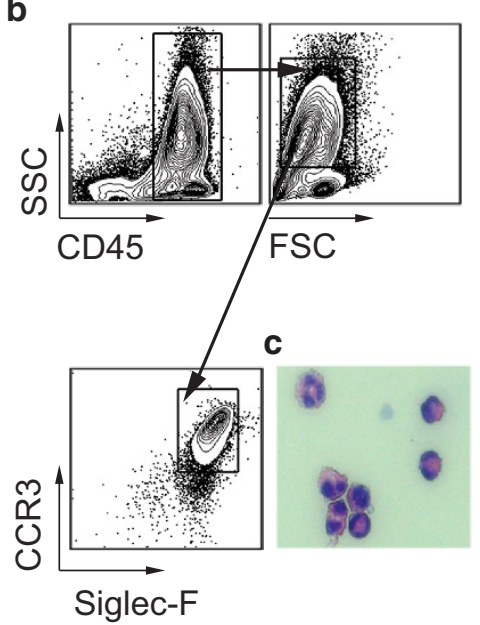

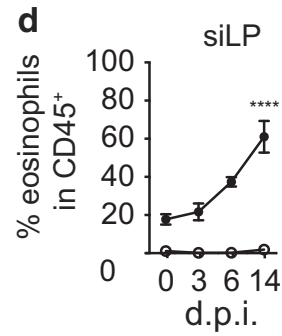

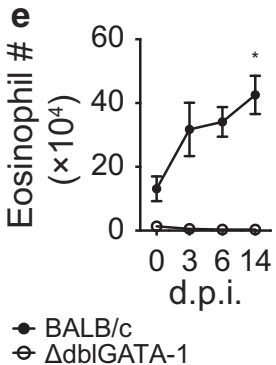

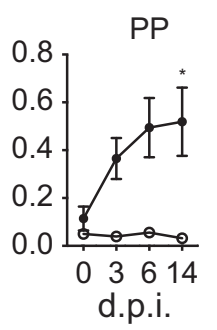
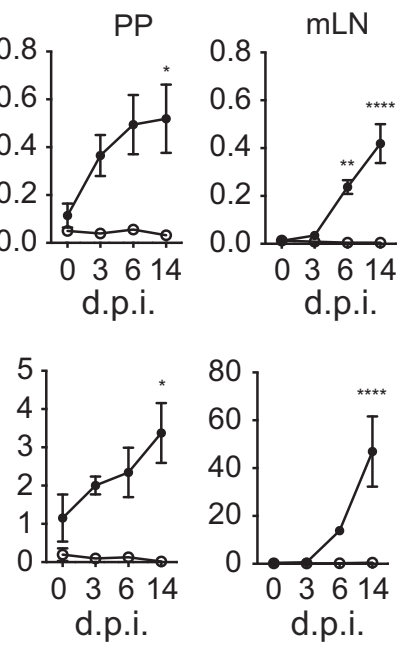

Figure 1 Accumulation of eosinophils in response to $H$. polygyrus infection. (a) Sirius red-stained eosinophils in sections from duodenum, Peyer's patches $(\mathrm{PP})$, and mesenteric lymph nodes $(\mathrm{mLN})$ of naive and $H$. polygyrus-infected (H.p.) WT mice. Arrowheads depict eosinophils. Scale bar $20 \mu \mathrm{m}$. (b) Gating strategy for the detection of eosinophils. (c) Morphology of FACS-sorted CD $45^{+} \mathrm{SSC}^{\text {hi }}$ Siglec-F ${ }^{+} \mathrm{CCR}^{+}$cells isolated from the small intestinal lamina propria (SiLP). (d) Frequencies of eosinophils in SiLP, PP, and mLN of WT (filled circles) and $\triangle$ dblGATA-1 mice (open circles) detected by flow cytometry. (e) Total numbers of eosinophils in SiLP, PP, and mLN. Mean \pm s.e.m. is shown. $n=3-4$. Data are representative of more than two independent experiments. Asterisks depict significant differences between naive and WT. ${ }^{\star} P<0.05,{ }^{\star \star} P<0.01,{ }^{\star \star \star \star} P<0.0001$. d.p.i.: days post infection.

restricted to $\mathrm{T}$ effector cells, we assessed the expression of GATA-3 in Foxp3 ${ }^{+}$regulatory T cells. Frequencies of GATA$3^{+}$Foxp $3^{+}$regulatory T cells increased similarly in the PP and $\mathrm{mLN}$ of both strains (Supplementary Figure S2F). In addition, the frequencies of T-bet ${ }^{+}$Th1 cells and IFN- $\gamma$-producing cells were unaffected by the absence of eosinophils (Supplementary Figure S2G, H).

To corroborate these results we repeated these experiments in infected PHIL mice, which lack eosinophils owing to their expression of a diphtera toxin transgene under the control of the eosinophil peroxidase promoter. ${ }^{23}$ In agreement with our findings in $\triangle$ dblGATA-1 mice we observed a shift toward GATA- ${ }^{+}{ }^{+} \mathrm{CD} 4{ }^{+} \mathrm{T}$ cells in the PP of PHIL mice 14 days post infection (Supplementary Figure S3A, B). Once again there was no difference in the Th2-type response when mLN from PHIL and C57BL/6 WT controls were compared (Supplementary Figure S3A, B). Similar to our findings in
$\Delta$ dblGATA-1 mice, and corresponding to previous findings, the cell number isolated from pooled PP of naive and infected PHIL mice were reduced compared with WT mice (Supplementary Figure S3C).

Eosinophil-deficient mice thus display altered T-cell responses during an enteric nematode infection, marked by a selective increase in $\mathrm{GATA}-3^{+} \mathrm{CD} 4{ }^{+}$Th2- and IL-4producing cells in the PP.

\section{Increased IL-4 production by TFH cells in infected eosinophil-deficient mice}

Follicular T helper cells (TFH) have been shown to be a major source of IL-4 in the mLN during helminth infections. ${ }^{24,25}$ We therefore assessed the phenotype of IL-4 producing $\mathrm{CD} 4{ }^{+} \mathrm{T}$ cells in further detail and used the expression of CXCR5 and PD- $1^{26}$ to define and quantify TFH cells in PP and $\mathrm{mLN}$. 

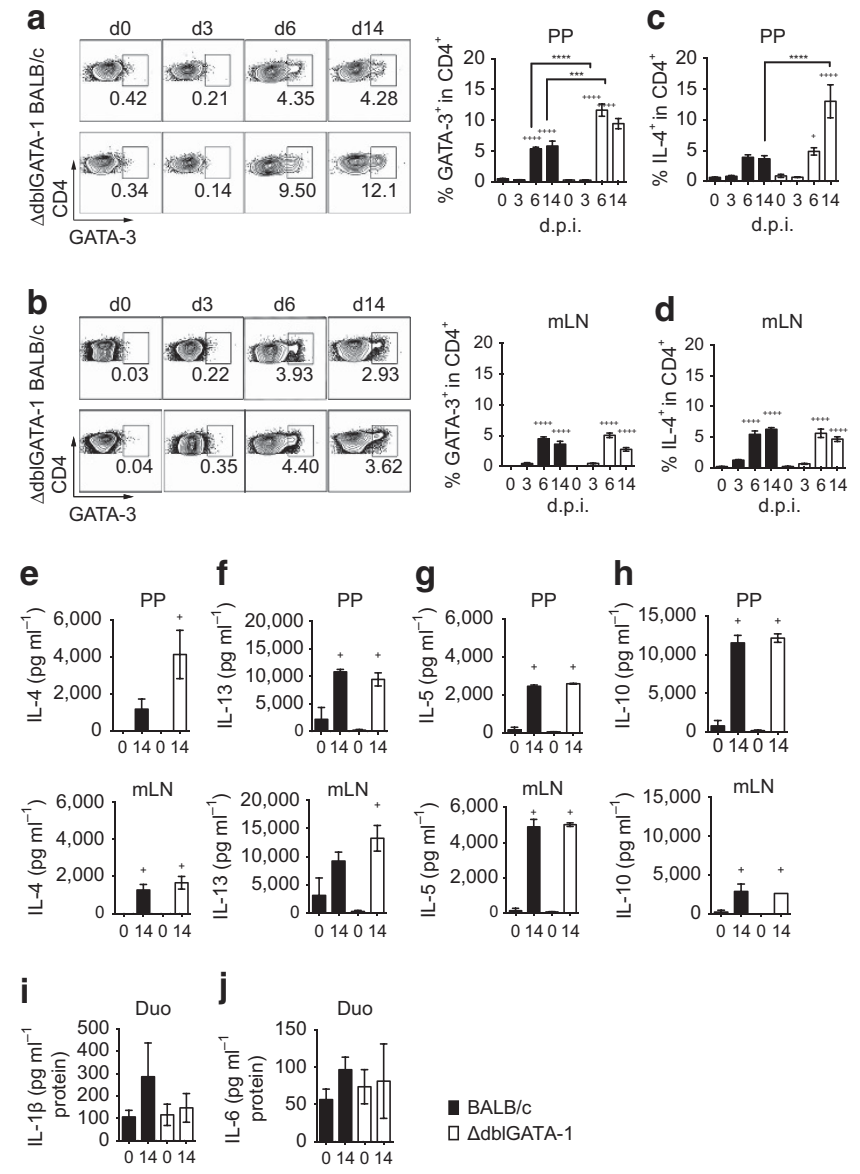

目 BALB/C

प $\triangle \mathrm{dbIGATA}-1$
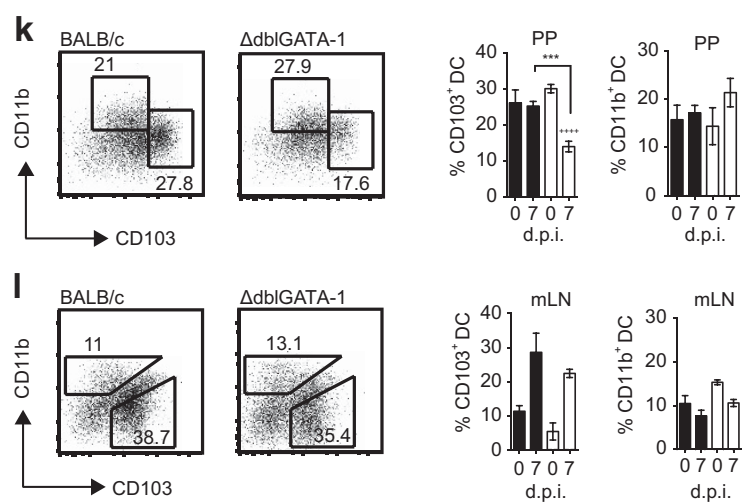

Figure 2 Preferential increase in GATA-3 ${ }^{+}$T cells and Th2 responses in $\mathrm{PP}$ of $H$. polygyrus-infected eosinophil-deficient mice. (a, b) Frequencies of GATA-3 ${ }^{+}$Th2 cells in live CD4 ${ }^{+}$cells isolated from PP (a) and $\mathrm{mLN}(\mathbf{b})$ of naive and $H$. polygyrus-infected WT (black bars) and $\Delta \mathrm{dbIGATA-1}$ mice (open bars). (c, d) Intracellular detection of IL-4 by $\mathrm{CD} 4{ }^{+}$cells isolated from PP (c) and $\mathrm{mLN}$ (d) and stimulated with PMA/ionomycin for $3 \mathrm{~h}$. (e-h) Secretion of IL-4, $-13,-5$, and -10 by PP and $\mathrm{mLN}$ cells stimulated for $72 \mathrm{~h}$ with anti-CD3/28 as measured by ELISA. (i, j) IL-1 $\beta$ and IL-6 in homogenized duodenum, normalized to total protein detected by BCA. $(\mathbf{k}, \mathrm{I})$ Representative FACS plots and frequencies of $\mathrm{CD}_{11 \mathrm{c}^{+}}$dendritic $^{-}$ cells expressing CD103 and CD11b in PP (k) and mLN (I). Mean \pm s.e.m. Data are representative of more than three independent experiments, $n=4-6(\mathbf{a}-\mathbf{d}, \mathbf{k}, \mathbf{I}), 1$ experiment, $n=4-5(\mathbf{e}-\mathbf{h})$ and two experiments, $n=3-5(\mathbf{i}, \mathbf{j}){ }^{* \star *} P<0.001,{ }^{* \star * \star} P<0.0001$. Level of significance comparing naive with infected groups is depicted by ${ }^{+}$.
As expected, the PP of naive animals displayed relatively high frequencies of $\mathrm{CXCR} 5^{+} \mathrm{PD}-1^{+} \mathrm{TFH}$ cells, permitting continuous IgA class switching at this site ${ }^{27}$ (Figure 3a). In contrast, few TFH cells were detected in the mLN of naive mice (Figure 3b). In response to infection, the number of CXCR5 ${ }^{+}$ PD- $1^{+}$cells in PP and mLN of WT and $\triangle$ dblGATA- 1 mice increased to a similar extent, with slightly lower frequencies observed in the PP of $\triangle$ dblGATA-1 compared with WT mice (Figure $3 \mathbf{a}$ and $\mathbf{b}$ ). We compared IL-4 production by CXCR5 ${ }^{+} \mathrm{PD}-1^{+}$TFH cells and CXCR5 ${ }^{-} \mathrm{PD}-1^{-}$non-TFH cells and found that both of these populations in the PP of infected $\Delta$ dblGATA-1 mice contained significantly higher frequencies of IL-4 producing cells compared with WT controls though the increase in IL-4-producing cells was greater in TFH cells (Figure 3c). Furthermore, on a per cell basis, TFH cells from $\Delta$ dblGATA-1 mice had significantly increased IL-4 production as indicated by a higher median fluorescence intensity, whereas non-TFH cells expressed similar levels of IL-4 in infected WT and $\triangle$ dblGATA-1 mice (Figure 3e). In contrast, except for a significant decrease of $\mathrm{IL}_{-} 4^{+}$non-TFH cells in mLN of $\Delta$ dblGATA-1 mice, the frequency of IL-4-expressing CD4 ${ }^{+}$ T cells, as well as IL- 4 amounts per cell were similar in WT and $\Delta$ dblGATA-1 mice (Figure 3d and $\mathbf{f}$ ).

To ensure that the increase in IL- $4^{+}$TFH cells was not compensatory for the slightly lower levels of TFH cells in the PP of $\Delta$ dblGATA- 1 mice, we further expressed IL- $4^{+}$TFH cells as a percentage of $\mathrm{CD} 4^{+}$cells, which were comparable between strains (Supplementary Figure S2C). Expressed in this way, the PP of $\Delta$ dblGATA-1 mice still contained significantly higher frequencies of IL-4 ${ }^{+}$TFH cells (Figure 3g). As the PP of $\triangle$ dblGATA-1 mice contain significantly fewer cells compared with BALB/c mice (Supplementary Figure S2B), this difference was not apparent when calculating total numbers of IL- $4^{+}$TFH cells (Figure $3 \mathbf{h}$ ).

IL-10 is a pleiotropic cytokine implicated in immune regulation and as a switch factor for $\operatorname{IgA} .^{28} \mathrm{~A}$ small percentage of TFH cells constitutively expressed IL-10 in PP and mLN, but this did not differ significantly between mouse strains and was not affected by $H$. polygyrus infection. A significant increase of IL-10 producing non-TFH cells was detected in $\mathrm{mLN}$ of both mouse strains upon $\mathrm{H}$. polygyrus-infection (Figure 3i-j).

We conclude that nematode infection in the absence of eosinophils leads to a deregulated $\mathrm{T}$-cell response in the $\mathrm{PP}$ with a prominent increase in IL-4 production by TFH cells.

\section{Eosinophil-deficient mice display increased class switching to IgG1}

Given that TFH-derived IL-4 supports IgG1 and IgE class switching ${ }^{25}$ we asked whether the increased availability of IL-4 in nematode-infected eosinophil-deficient mice affected germinal centre formation and class switching to Th2-associated antibodies following nematode infection.

Indeed, serum levels of total IgG1 and IgE were significantly increased in eosinophil-deficient mice acutely infected with nematodes (Figure 4a and $\mathbf{b}$ ). In contrast, levels of circulating IgG2a were not affected by $H$. polygyrus infection and did not 
a

$\mathrm{PP} \mathrm{CD4} 4^{+}$
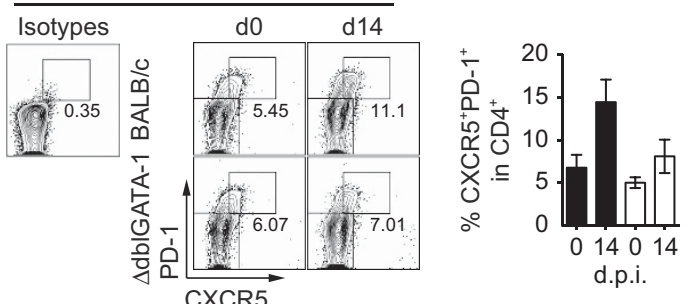

b $\mathrm{mLN} \mathrm{CD4}^{+}$

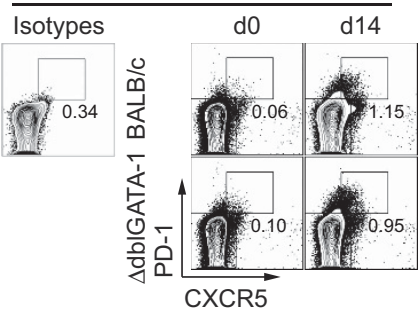

BALB/c

$\square \triangle \mathrm{dbIGATA}-1$

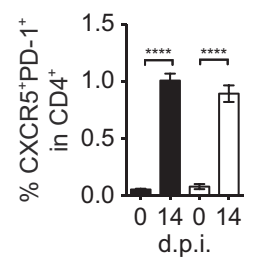

C PP CD4 $4^{+} \mathrm{d} 14$
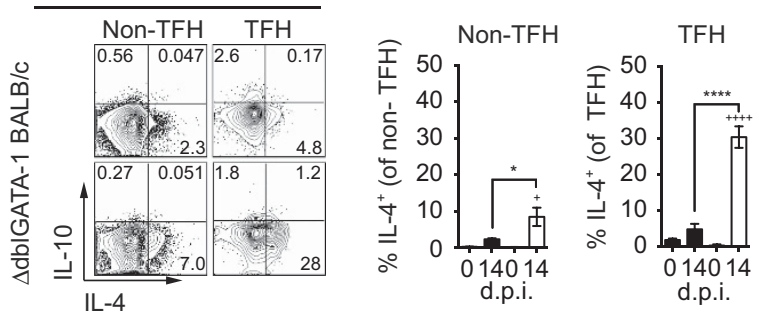

e
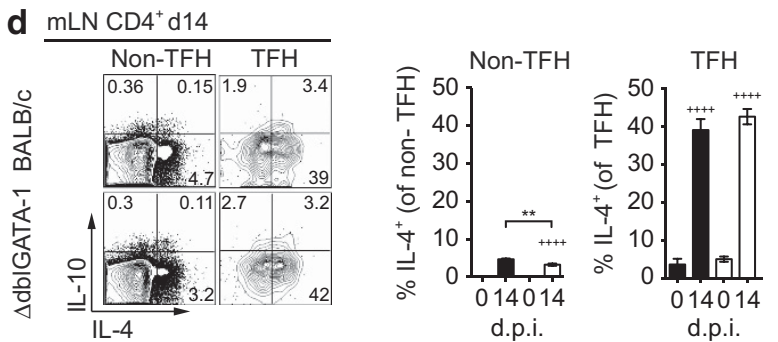

f
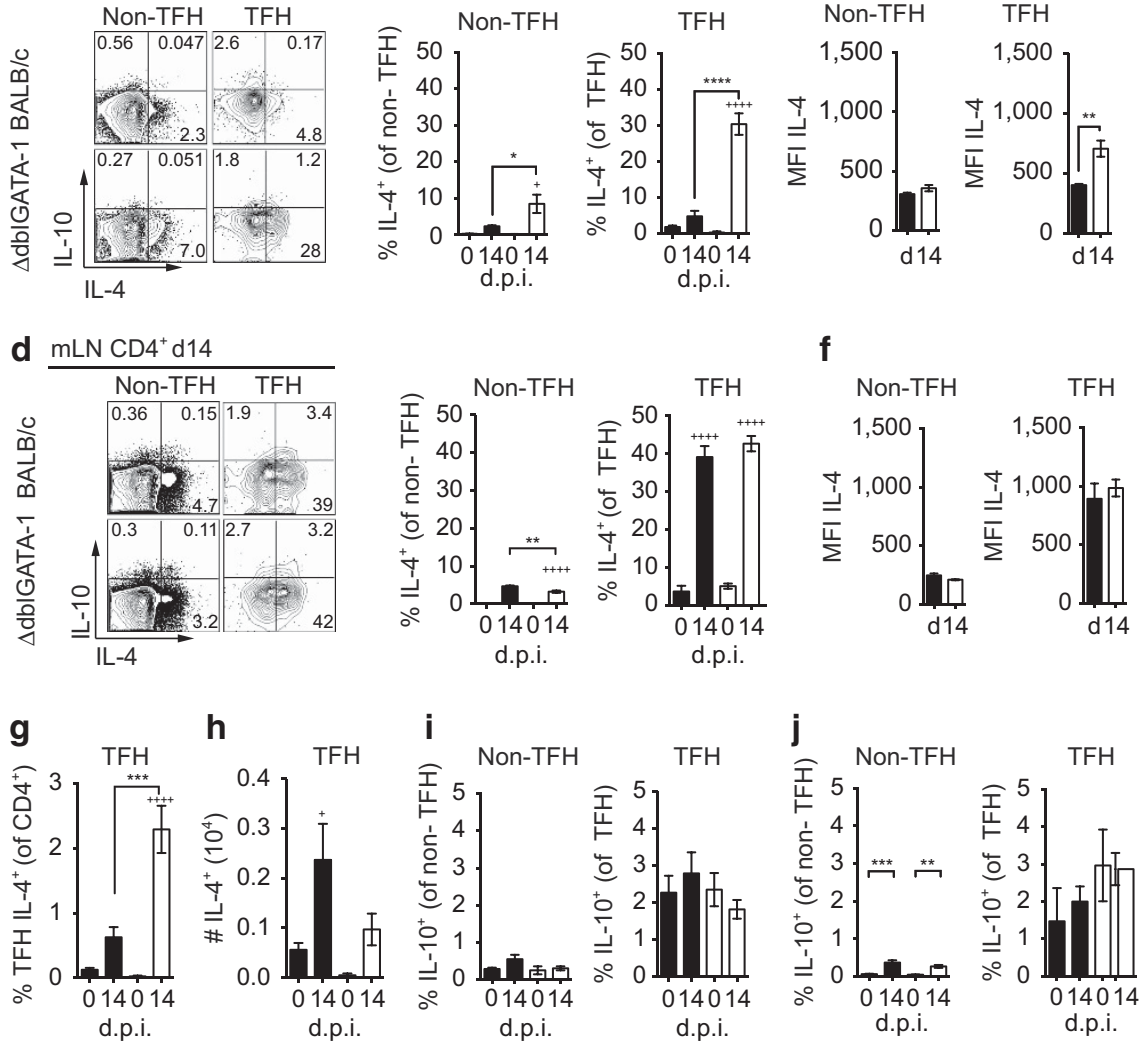

Figure 3 Increased IL-4 production by TFH cells in PP of eosinophil-deficient mice infected with $H$. polygyrus. (a, b) Exemplary FACS plots and frequencies of CXCR5 and PD-1 expression by PP CD4 ${ }^{+} \mathrm{T}$ cells $(\mathbf{a})$ and $\mathrm{mLN}(\mathbf{b}) \mathrm{CD} 4^{+}$cells from naive and $H$. polygyrus-infected WT (black bars) and $\triangle$ dbIGATA-1 mice (open bars). (c, d) Exemplary FACS plots and frequencies of IL- $4^{+}$CD4 ${ }^{+}$CXCR5 ${ }^{-}$PD- $1^{-}$non-TFH and CD4 ${ }^{+}$CXCR5 ${ }^{+}$PD- $1^{+}$TFH cells from $\mathrm{PP}(\mathbf{c})$ and $\mathrm{mLN}(\mathbf{d})$ stimulated with PMA/ionomycin for $3 \mathrm{~h}$ at day 14 p.i. (e, f) IL-4 median fluorescence intensities detected for non-TFH and TFH cells from PP (e) and mLN (f). (g) IL-4 + TFH cells expressed as frequencies of total CD4 ${ }^{+}$cells in PP. (h) Total number of PP IL- $4^{+}$TFH cells. (i, j) Quantification of IL-10+ $10^{+}$non-TFH and TFH cells in the PP (i) and $\mathrm{mLN}$ (j). Mean \pm s.e.m. $n=4-5$. Data are representative of four independent experiments. ${ }^{*} P<0.05,{ }^{* * *}<0.001,{ }^{* * * *} P<0.0001$. Level of significance comparing naive with infected groups is depicted by ${ }^{+}$.

differ between mouse strains (Figure 4c). Serum levels of IgA were similar in BALB/c and $\Delta$ dblGATA-1 mice (Figure 4 d), but naive $\Delta$ dblGATA-1 mice displayed significantly reduced levels of intestinal IgA (Figure 4e), confirming previous studies. ${ }^{16,19}$ In line with total serum IgG1 and IgE, the serum of $\Delta$ dblGATA-1 mice exposed to a challenge infection displayed significantly higher titers of IgG1 and IgE antibodies recognizing $H$. polygyrus excreted/secreted products than did serum 

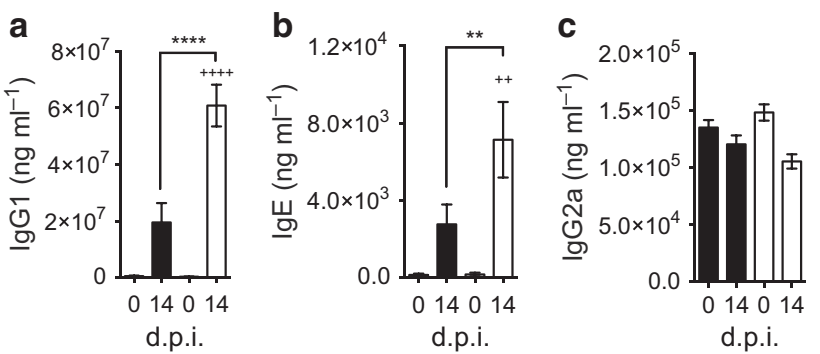

$\mathrm{BALB} / \mathrm{C}$

प $\triangle \mathrm{dbIGATA}-1$

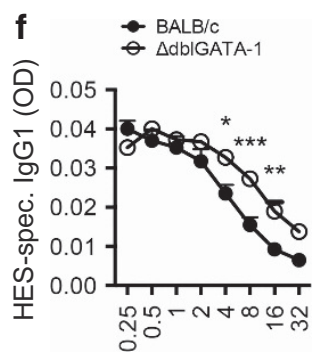

Fold dilution $\left(\mathrm{x} 10^{2}\right)$

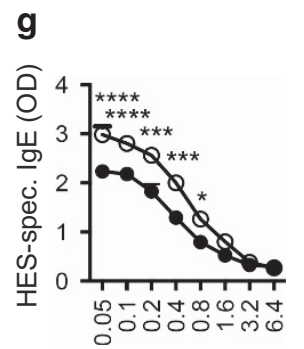

Fold dilution $\left(\times 10^{2}\right)$

d.p.i.
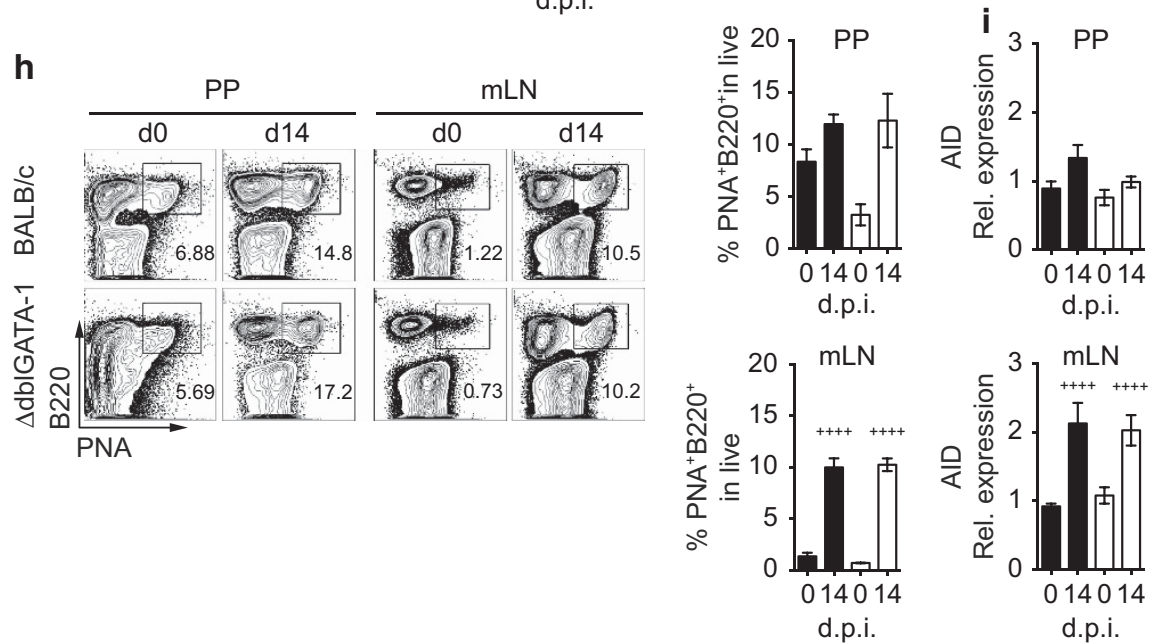

Figure 4 Increased IgG1 and IgE responses and normal GC formation in H. polygyrus-infected eosinophil-deficient mice. (a-d) Levels of IgG1 (a), IgE (b), IgG2a (c) and IgA (d) in serum of naive and H. polygyrus-infected WT (black bars) and $\triangle$ dbIGATA-1 mice (open bars). (e) Small intestinal IgA detected in $2 \mathrm{~cm}$ duodenal organ cultures. (f, $\mathbf{g})$ Titration of $H$. polygyrus excretory/secretory antigen-(HES)-specific lgG1 and lgE in serum of WT (filled circles) and $\triangle \mathrm{dbIGATA}-1$ mice (open circles) at day 11 post challenge infection. (h) Exemplary FACS plots and frequencies showing PNA ${ }^{\text {hi }}$ B220 $^{+}$germinal centre (GC) B cells in PP and mLN of naive and $H$. polygyrus-infected WT and $\triangle \mathrm{db}$ IGATA-1 mice. (i) Expression of activation-induced cytidine deaminase (AID) mRNA in PP and $\mathrm{mLN}$ tissue. Mean \pm s.e.m. is shown. Data in a-e are pooled from two independent experiments with $3-6$ animals each. Data in $\mathbf{f}-\mathbf{i}$ are representative of more than three independent experiments. $n=3-5$. ${ }^{\star} P<0.05,{ }^{* \star} P<0.01$, ${ }^{\star * \star} P<0.001$ and ${ }^{* * *} P<0.0001$. Level of significance comparing naive with infected groups is depicted by ${ }^{+}$.

from challenged WT controls (Figure $4 \mathbf{f}$ and $\mathbf{g}$ ). To determine whether differences in serum antibody levels were due to altered germinal center (GC) properties, we next analyzed B cells in PP and mLN-the two major sites for gut-associated B-cell class switching.

In accordance with the prominent population of TFH cells at baseline, the PP of naive mice contained relatively high levels of $\mathrm{PNA}^{\text {hi }} \mathrm{B}_{22}{ }^{+}$germinal centre B cells. Slightly lower baseline frequencies of $\mathrm{PNA}^{\text {hi }} \mathrm{B} 220^{+}$cells were seen in the PP of naive $\Delta$ dblGATA-1 mice compared with naive WT controls, but this difference did not reach statistical significance. 14 days post infection with $H$. polygyrus there was only a moderate increase in the frequency of GC B cells in both mouse strains. Once again the situation in the $\mathrm{mLN}$ was quite different. Here GC B cells were almost absent in naive mice, but increased significantly and to a similar extent upon infection in both mouse strains (Figure 4h).

Assessing the expression of activation-induced cytidine deaminase, an enzyme required for somatic hyper-mutation and isotype class switching that is confined mainly to GC B cells, ${ }^{29}$ we confirmed that $H$. polygyrus infection only moderately increased class switch recombination in the PP, but 
resulted in significant upregulation of activation-induced cytidine deaminase in the $\mathrm{mLN}$ of infected WT and eosinophil-deficient mice (Figure 4i). Hence, GC formation in response to infection was intact in $\mathrm{PP}$ and $\mathrm{mLN}$ of eosinophil-deficient mice.

Next we assessed the expression of antibody isotypes by GC B cells in the PP and mLN. WT mice only moderately increased IgG1 and expanded IgA production in the PP in response to infection, indicating that the PP are not the main site of IgG1 responses to $H$. polygyrus in these animals (Figure $\mathbf{5 a}$ and $\mathbf{b}$ ). In contrast, PP of $\Delta$ dblGATA-1 mice displayed markedly increased frequencies of IgG1 ${ }^{+}$GC B cells (Figure $5 \mathbf{a}$ and $\mathbf{b}$ ) and a sharp decline of $\operatorname{IgA}^{+}$B-cell frequencies and numbers (Figure 5c and d) compared with infected WT controls. In $\mathrm{mLN}$, the infection led to a considerable and similar increase in IgG1-switched GC B cells in WT and eosinophil-deficient mice (Figure 5e and $\mathbf{f}$ ), whereas the small $\operatorname{IgA}^{+}$B-cell population decreased in frequency, but was maintained in terms of absolute numbers following infection in both strains (Figure $\mathbf{5 g}$ and $\mathbf{h}$ ). As TGF- $\beta$ is of imperative importance for IgA class switching, ${ }^{28}$ and as eosinophils are known to produce TGF- $\beta^{30}$ we compared the gene expression of TGF- $\beta$ in the small intestine early in infection and quantified TGF- $\beta$ protein in intestinal tissue at day 14 post infections. Neither TGF- $\beta$ expression nor levels of TGF- $\beta$ protein were reduced in eosinophil-deficient mice (Figure $5 \mathbf{i}$ and $\mathbf{j}$ ).

Hence, we conclude that during intestinal nematode infection, IgG1 switching takes place predominantly in the $\mathrm{mLN}$, which is not affected by eosinophil deficiency. In the PP, however, eosinophils are required to limit class switch recombination to IgG1 and thus are crucial for the maintenance of IgA production.

Importantly, as PP do not increase in size following H. polygyrus infection (Supplementary Figure S2B), the increased frequency of $\mathrm{IgG1}^{+}$B cells in PP of infected $\Delta$ dblGATA-1 mice is unlikely to represent a difference in infiltrating cells. Rather, eosinophils influence the flavor of PP antibody production, maintaining IgA class switch recombination, whereas their absence leads to enhanced class switch recombination to IgG1 and increased serum IgG1 levels.

\section{Eosinophil reconstitution does not lead to restricted Th2 responses in the PP of $\Delta \mathrm{dbIGATA}-1$ mice}

To assess whether the finding of elevated Th2 induction and IgG1 class switching in PP of infected $\Delta$ dblGATA-1 mice could
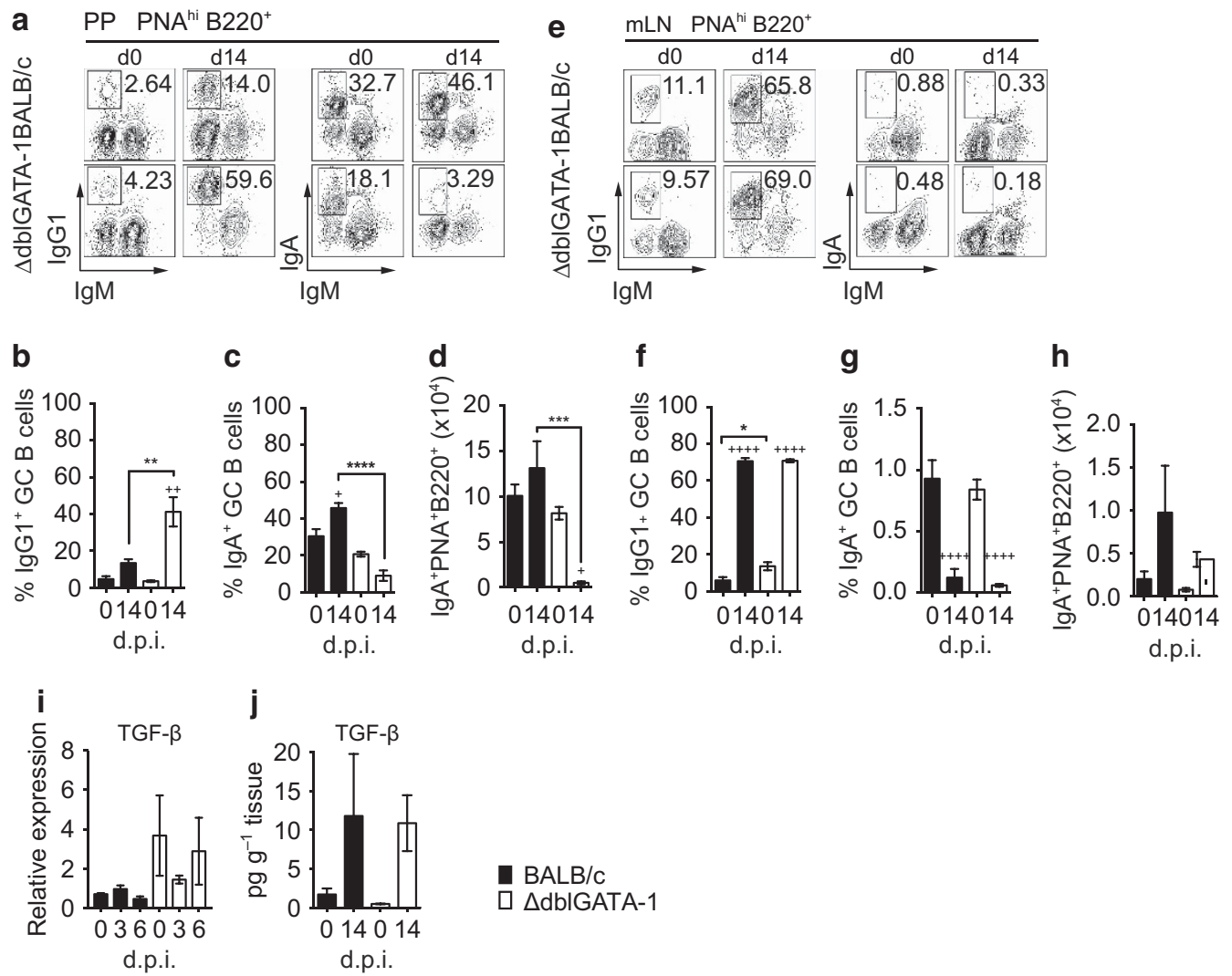

Figure 5 Increased IgG1 class switching during infection is restricted to Peyer's patches in eosinophil-deficient $\triangle$ dblGATA-1 mice. (a) Exemplary FACS plots of IgG1, IgM, and IgA expression by PNA ${ }^{\text {hiB } 220^{+}}$GC B cells in PP of naive and $H$. polygyrus-infected WT and $\triangle \mathrm{dblGATA}-1$ mice. (b, c) Frequencies of $\operatorname{lgG} 1^{+}$(b) and IgA ${ }^{+}$(c) GC B cells in PP of naive and $H$. polygyrus infected WT (black bars) and $\triangle \mathrm{dblGATA}-1$ mice (open bars). (d) Absolute numbers of IgA ${ }^{+}$CG B cells in PP. (e-h) Exemplary FACS plots (e), frequencies of $\operatorname{lgG} 1^{+}$(f), and IgA ${ }^{+} \mathrm{GC} \mathrm{B}$ cells (g) and total numbers of IgA ${ }^{+}$GC B cells (h) in mLN of naive and infected WT and $\Delta$ dblGATA-1 mice. (i) Relative expression of TGF- $\beta$ day $0-6$ days post infection (d.p.i.). (j) Levels of TGF- $\beta$ protein in supernatants of duodenal organ cultures, normalized to tissue weight. Mean \pm s.e.m. is shown. $n=3-5$. Data are representative of at least three $(\mathbf{a}-\mathbf{h})$ or $2(\mathbf{i}, \mathbf{j})$ independent experiments. ${ }^{\star} P<0.05,{ }^{\star \star} P<0.01,{ }^{\star \star \star} P<0.001,{ }^{\star \star \star \star} P<0.0001$. Level of significance comparing naive with infected groups is depicted by ${ }^{+}$. 
be controlled by eosinophil reconstitution, we isolated eosinophils from the blood of IL-5 transgenic mice and performed intravaneous transfers of $5-10 \times 10^{6}$ cells prior to and during $H$. polygyrus infection. A scheme of the approach used and the purity of transferred eosinophils after enrichment are shown in Supplementary Figure S4A and B. Transferred eosinophils were prominent in the lamina propria of $\Delta$ dblGATA-1 mice 7 days post $H$. polygyrus infection, but was substantially lower than equivalent frequencies in infected WT mice (Supplementary Figure S4C). However, PP cellularity was not restored as a result of eosinophil transfer to $\Delta$ dblGATA-1 mice (Supplementary Figure S4D), nor did the presence of eosinophils in the lamina propria of $\triangle$ dblGATA-1 mice restrict the induction of PP GATA- ${ }^{+}$Th2 cells to levels of BALB/c mice (Supplementary Figure S4E). Similarly, the PP of $\triangle$ dblGATA-1 mice displayed significantly reduced frequencies of $\operatorname{IgA}^{+} \mathrm{B}$ cells and a trend for higher frequencies of IgG1 B cells following eosinophil transfers (Supplementary Figure S4F, G).

Hence, the reconstitution of $\Delta$ dblGATA-1 mice with bloodderived eosinophils was insufficient in preventing the overt Th2 induction and IgG1 class switching in PP.

Parasite fecundity is impaired in eosinophil-deficient mice The Th2-type response and the associated class switch to IgG1 in response to $H$. polygyrus infection are central for the defence against re-infection, and for the control of parasite fecundity in primary infections. ${ }^{31}$ We therefore asked whether the marked increase in PP Th2 reactivity and IgG1 production in the absence of eosinophils affects worm burden and fitness. There was no difference in adult worm burdens at 2 weeks post infection in $\triangle$ dblGATA-1 and PHIL mice compared with WT controls, suggesting that the development of L3 larvae to adults, as well as their emergence to the lumen is not affected by the absence of eosinophils (Figure 6a and c). Protective immunity to challenge infection was also not affected by the lack of eosinophils (Figure 6a). However, quantifying egg production by female worms isolated at 2 weeks post infection revealed that parasite fecundity, a measure of fitness, was significantly reduced in both eosinophil-deficient mouse strains compared with WT controls (Figure $\mathbf{6 b}$ and $\mathbf{d}$ ).

In response to IL- $4 \mathrm{R} \alpha$ signaling, macrophages acquire an alternatively activated phenotype marked by the expression of resistin-like molecule-alpha (Relm- $\alpha$ ) and arginase- $1(\arg -1),{ }^{32}$ the latter being central to the control of challenge infection with H. polygyrus. ${ }^{33}$ We therefore asked whether the decreased parasite fitness during primary $H$. polygyrus infections in the absence of eosinophils was related to changes in alternative macrophage activation. The expression of Relm- $\alpha$ and arginase-1 in small intestinal tissue, however, was similar in infected WT and $\Delta$ dblGATA-1 mice (Figure $6 \mathbf{e}$ and $\mathbf{f}$ ).

In response to IL-4 and -13, intestinal epithelial cells differentiate into goblet cells that produce resistin-like molecule beta (Relm- $\beta$ ) - a factor impeding intestinal nematodes in feeding from host tissue. ${ }^{34}$ It was thus possible that differences in Relm- $\beta$ production or intestinal goblet cell numbers could account for the decreased fecundity of female worms. However, we found that small intestinal Relm- $\beta$ expression and goblet cell numbers were comparable in infected WT and $\Delta$ dblGATA-1 mice (Figure 6g-i). Interestingly, despite similar goblet cell counts detected in infected WT and $\Delta$ dblGATA-1 mice, we found that the mucus layer attached to small intestinal villi of infected WT mice was practically absent in infected eosinophildeficient mice (Figure 6h).

Finally, we assessed the level of infection-induced small intestinal enteropathy at day 3 and 14 post infection and found that both WT and eosinophil-deficient mice developed similar enteritis scores in response to infection (Figure 6j).

Taken together, these data indicate that the action of eosinophils in $H$. polygyrus infection is not detrimental for parasite development during primary infections and dispensable for protection against challenge infections. Instead, they rather regulate the development of Th2-type responses associated with IgG1 production in the PP, presumably in order to maintain a balanced mucosal antibody profile.

\section{DISCUSSION}

This study demonstrates a novel role for eosinophils in regulating adaptive immune responses in the PP compartment of the gut following small intestinal nematode infection. Previous studies have shown that at steady state eosinophils are centrally involved in supporting PP development as well as in sustaining IgA class switch recombination at this site. ${ }^{16,19}$

Our study builds on these findings by showing that deregulation of B-cell responses in the absence of eosinophils extends to pathogen-induced IgG1 responses. In addition, this study demonstrates a novel role for eosinophils in regulating T-cell function in PP upon enteric infection.

During lung inflammation eosinophils support the priming and accumulation of Th2 cells ${ }^{8,35}$ and during an oral sensitization regime, using peanut allergen combined with the mucosal adjuvant cholera toxin, eosinopohils have been shown to be critically involved in the induction of systemic Th2 responses. ${ }^{36}$ Our data, however, show that Th2 induction in the mLN's in responses to infection with a natural enteric parasite were intact in two strains of eosinophil-deficient mice and that the absence of eosinophils leads to a significant increase in GATA-3-expressing and IL-4 producing Th2 cells in the PP. Thus, we show that eosinophils are not a prerequisite for proper Th2 induction in response to intestinal pathogens and that they clearly vary in their propensity to support and regulate Th2 responses under different conditions and in different compartments.

The effect of eosinophil deficiency on immune responses in the PP is unlikely to be a direct one as, although they do infiltrate the PP following $H$. polygyrus infection, their numbers are extremely low. In contrast, eosinophils infiltrate the lamina propria in vast numbers. Thus, eosinophils are likely to exert their effect(s) in the lamina propria, ultimately influencing events in the PP. In our study, frequencies of $\mathrm{CD} 103^{+} \mathrm{CD} 11 \mathrm{~b}$ DC were found to be higher in the PP, but not the mLN, of $\mathrm{BALB} / \mathrm{c}$ mice, as compared with $\triangle$ dblGATA-1 animals, following infection with $H$. polygyrus. This subset has been 

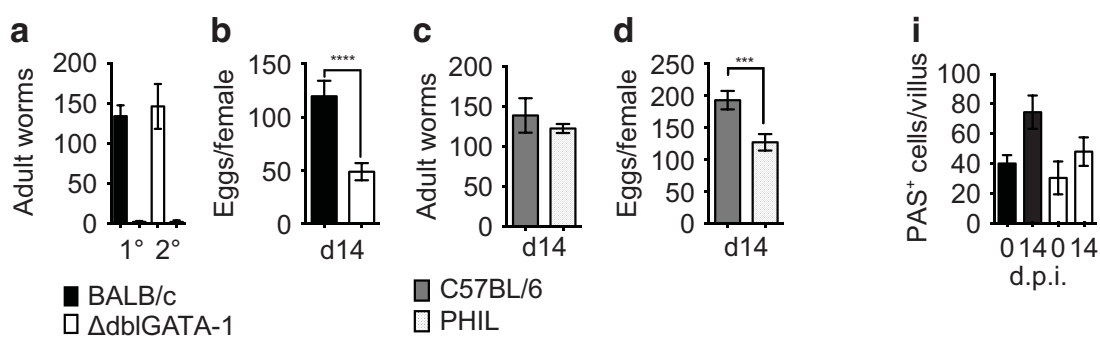

BALB/c
BdbIGATA-1

$\square$ PHIL
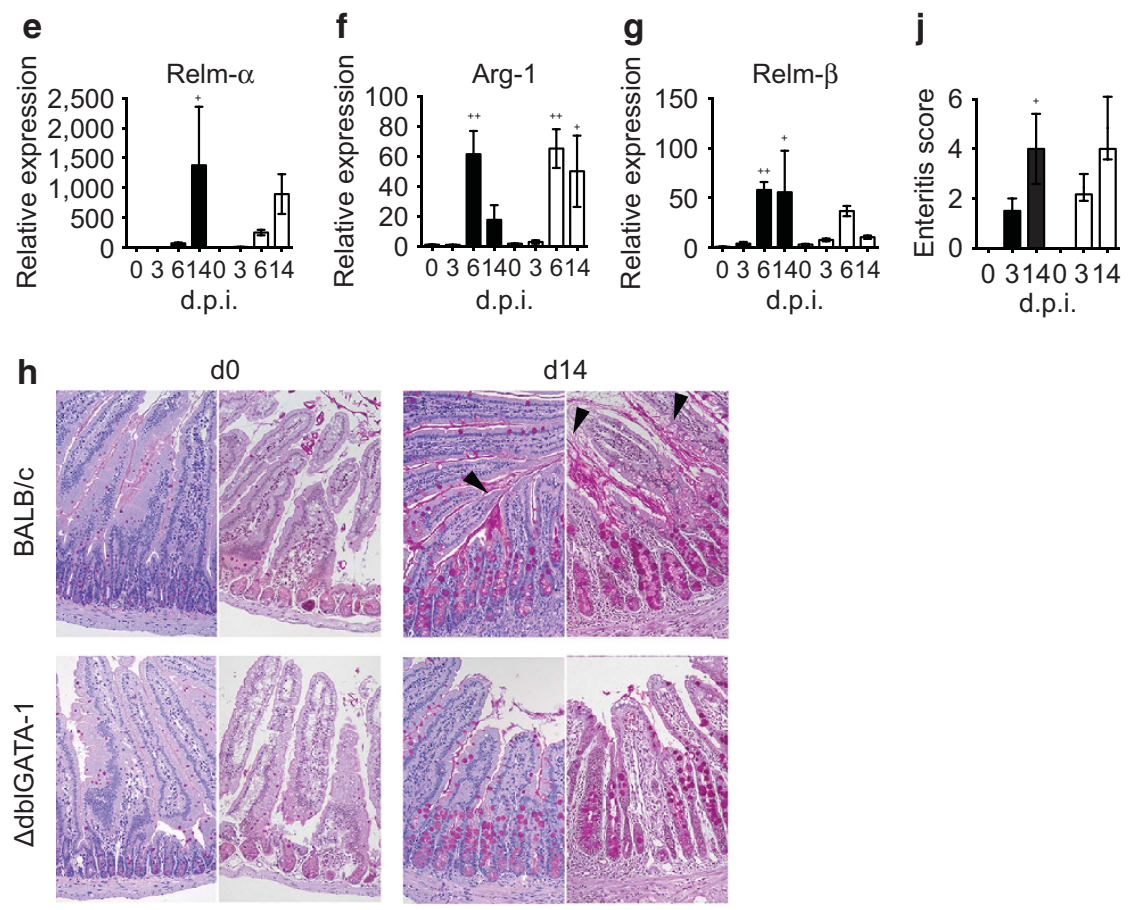

Figure 6 Parasite fecundity is impaired in the absence of eosinophils. (a) Adult worm burden in WT (black bars) and $\Delta$ dblGATA-1 mice (open bars) at day 14 post primary and day 11 post challenge $H$. polygyrus infection. (b) Individual fecundity of female worms isolated at day 14 after primary $H$. polygyrus infection from WT BALB/c and $\Delta$ dbIGATA-1 mice. (c) Adult worm burden in WT C57BL/6 (dark gray bars) and PHIL mice (light gray) at day 14 post primary infection. (d) Fecundity of female worms isolated from C57BL/6 and PHIL mice. (e-g) Relm- $\alpha$ (e), Arginase-1 (Arg-1) (f) and Relm- $\beta$ (g) mRNA expression in duodenal tissues of naive and infected WT and $\Delta \mathrm{dblGATA-1}$ mice. (h) Duodenum cross sections from BALB/c and $\Delta \mathrm{dblGATA-1}$ mice at day 14 post primary $H$. polygyrus infection stained with periodic acid/Schiff (PAS) for goblet cell quantification. Black arrowheads mark areas of extensive mucus deposition. Magnification $\times 100$. Two representative pictures per time point and mouse strain from two independent experiments are shown. (i) Quantification of PAS ${ }^{+}$cells in naive and infected BALB/c and $\triangle \mathrm{dbIGATA-1}$ mice. (j) Enteritis score of duodenum in naive and infected BALB/c and $\Delta$ dbIGATA-1 mice. Mean \pm s.e.m. $n=3-5$. Data from BALB/c and $\Delta \mathrm{db}$ IGATA-1 mice are representative of three independent experiments. Experiment with C57BL/6 and PHIL mice was performed once. Significance tested by Mann-Whitney $U$-test (a-d) and one-way ANOVA with HolmSidaks multiple comparison test $(\mathbf{e}-\mathbf{k})$. ${ }^{\star} P<0.05,{ }^{\star \star} P<0.01,{ }^{\star \star *} P<0.001{ }^{\star * *} P<0.0001$. Level of significance comparing naive with infected groups is depicted by ${ }^{+}$.

implicated in regulation of Th2 responses via the production of IL-12. ${ }^{22}$ It is thus possible that eosinophil-mediated support of $\mathrm{CD} 103^{+} \mathrm{CD}_{11 \mathrm{~b}}{ }^{-} \mathrm{DC}$ provide conditions limiting Th2 differentiation in the PP.

Factors responsible for the maintenance of DC populations and their migration between intestine and gut-associated lymphoid organs are not well defined and studies are usually confined to assessing the effect of different stimuli in vitro on bone marrow derived DC. In this way, it has been shown that IL-1 $\beta$ induces the production of DC-derived IL- $12 .{ }^{37} \mathrm{IL}-1 \beta$ produced by eosinophils has further been implicated as responsible for their role in supporting intestinal $\operatorname{IgA}^{19}$ and intestinal IL- $1 \beta$ has been shown to limit Th2 responses following $H$. polygyrus infection. ${ }^{21}$ Small intestinal IL-1 $\beta$ levels of $H$. polygyrus-infected BALB/c and $\Delta$ dblGATA-1 mice, however, were similar, suggesting that the differences seen in

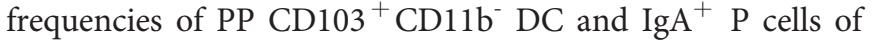
$\mathrm{BALB} / \mathrm{c}$ and $\Delta$ dblGATA-1 mice occur independently of IL-1 $\beta$.

In our study, transfers of eosinophils from IL-5 transgenic mice did not result in a restriction of PP Th2 responses of $\Delta$ dblGATA-1 mice comparable to the levels seen in WT mice. There are several possible explanations for this. The first possibility is that the long-term presence of eosinophils is important for PP development and that our relatively short protocol (starting 8 days pre-infection) was insufficient for the establishment of a fully "eosinophil competent" environment. Second, it is possible that the numbers of eosinophils infiltrating the gut after transfers, being reduced by $\sim 50 \%$ 
compared with WT mice, may have been insufficient for controlling the immune reactions in PP. Furthermore, eosinophils derived from IL-5 transgenic mice may be distinct from eosinophils found in the healthy intestine. IL-5 is essentially an inflammatory cytokine and responsible for eosinophil infiltration to sites of infection and inflammation. In contrast, homeostatic trafficking of eosinophils is regulated by eotaxin. ${ }^{10}$ Thus, eosinophils infiltrating the gut in response to $H$. polygyrus infection in WT mice (and the IL-5-elicited eosinophils transferred in our study) may differ in their actions from eosinophils present in high numbers in the small intestine of healthy mice. In accordance, it has been shown that activated Siglec- $\mathrm{F}^{\text {hi }}$ eosinophils infiltrate the intestine in high numbers during experimental colitis and express the degranulation marker CD63. ${ }^{38}$ In contrast healthy mice have a steady population of Siglec- $\mathrm{F}^{\text {int }}$, non-degranulating eosinophils. ${ }^{38}$ In the light of this, transfers of lamina propria eosinophils from healthy mice may yield better insight into the influence of eosinophils on PP immune responses.

It has previously been shown that the microbiota of eosinophil-deficient mice differs considerably to that of WT controls $^{16,19}$ and a recent review discusses the potential of eosinophils as regulators of the microbiome. ${ }^{39} \mathrm{PP}$ development is intimately linked to the presence of intestinal microbes and it is thus possible that PP immune responses are differentially regulated as a result of distinctions in the microbiome in the absence of eosinophils.

However, two previous studies in which a decrease in PP cellularity has been observed in eosinophil-deficient mice may suggest that this is a stable phenotype of eosinophil deficiency. Whereas Chu et al. ${ }^{16}$ report that $\Delta$ dblGATA-1 animals harbor higher intestinal levels of bacteroides and lower levels of firmicutes than $\mathrm{BALB} / \mathrm{c}$ mice, Jung et al. ${ }^{19}$ report the opposite observation. Despite this, both studies demonstrate a clear reduction in PP cellularity of $\triangle$ dblGATA-1 mice, as well as diminished intestinal levels of IgA. Nevertheless, it remains possible that distinctions in the microbiota as a result of eosinophil deficiency may contribute to enhanced Th2 immunity in the PP. Future work will examine this exciting prospect.

In our study, significantly enhanced IL-4 production was especially apparent in TFH cells of nematode-infected, eosinophil-deficient mice. TFH cells are essential in supporting B-cell class switch recombination and affinity maturation ${ }^{40}$ and following helminth infection, TFH cells increase in draining lymph nodes and produce high levels of IL-4 that promotes class switching to IgG1 and IgE. ${ }^{24,25}$ The PP, on the other hand, are the main location for induction of $\operatorname{IgA}^{+} \mathrm{B}$ cells. ${ }^{27}$

Previous studies have shown that eosinophils support B-cell survival $^{15}$ and IgA class switching in the intestine under homeostatic conditions. ${ }^{16,19}$ As B-cell class switching to IgA almost completely ceased in the PP of eosinophil-deficient mice following infection with $H$. polygyrus, our study suggests that eosinophils are also critically needed for the maintenance of the $\mathrm{PP}$ as a main site for IgA class switching during infection with an enteric pathogen. Furthermore, we found that the PP was not a major site for IgG1 class switching in response to $H$. polygyrus infection in WT mice. Thus, as $\Delta$ dblGATA-1 displayed frequencies of $\mathrm{IgG1}{ }^{+} \mathrm{GC}$ B cells in the PP almost comparable to the $\mathrm{mLN}$, our data point out the importance of eosinophils for the compartmentalization of antibody responses in distinct gut-associated lymphoid tissues. Of note, IL-4 has been shown to directly limit IgA class switching of naive human B cells. ${ }^{41}$ Thus, the increased IL-4 production by TFH cells detected in our study may, in addition to supporting IgG1 class switching, contribute directly to the loss of IgA.

Antibodies have an important role in protective immunity against challenge infections with $H$. polygyrus but are not thought to have a major role in worm expulsion during primary infections. ${ }^{31}$ However, mice lacking functional B cells $\left(\mathrm{J}_{\mathrm{H}}^{-1-}\right)$ shed significantly more eggs in the feces during a primary infection compared with WT mice and transfer of naive sera is sufficient to decrease egg shedding. This suggests that natural antibodies, as well as other serum components such as complement might have a role in reducing parasite fecundity. ${ }^{31}$ Thus, it is possible that the increase in serum IgG1 seen in the absence of eosinophils contribute to the observed impaired fecundity of parasites developing in $\triangle$ dblGATA-1 mice.

Confirming previous studies reporting on a defective mucous shield in the intestine in absence of eosinophils, ${ }^{16,19}$ we found that the mucus layer developing in WT mice in response to $H$. polygyrus infection was virtually absent in infected eosinophildeficient mice. It has been previously suggested that $H$. polygyrus feeds on host epithelial cells. ${ }^{42}$ If the mucus layer constitutes an additional nutrient source for $\mathrm{H}$. polygyrus, it is possible that resource limitation is responsible for the decreased fecundity seen in worms developing in $\triangle$ dblGATA-1 mice. Of note, numbers of mucus producing goblet cells as well as expression levels of the goblet cell-derived anti-nematode effector molecule Relm- $\beta^{34}$ were similar in both strains.

In summary, our data suggest that the gut-draining mLNs are the principal induction site for Th2 cytokines and associated IgG1 antibody responses to $H$. polygyrus infection, and that these responses are unaffected by eosinophil deficiency. Upon nematode infection, eosinophils are, however, essential for the regulation of Th2 -associated responses in the PP. They prevent excessive IL- 4 and IgG1 production in the PP, thereby assuring the maintenance of IgA class switching, presumably to protect the gut from bacterial invasion. Thus, our data show a novel regulatory role for eosinophils in guarding the compartmentalized immune response upon infection with an intestinal pathogen.

\section{MATERIALS AND METHODS}

Mice, infections, and parasite fecundity. $\triangle$ dblGATA-1 mice (Jackson Laboratory, CA, USA) were bred at the Institute of Immunology, Freie Universität Berlin. PHIL mice were bred at the German Arthritis Research Center, Berlin. Age and sex matched BALB/c and C57BL/6 WT mice were purchased from Janvier Labs (Saint-Berthevin, France). IL-5 transgenic NJ.1638 were a kind gift from Professor D. Vöhringer, Universitätsklinikum, Erlangen. All experiments were performed in accordance with the National Animal Protection Guidelines and approved by the German Animal Ethics Committee for the protection of animals (GO113/15). 
H. polygyrus was maintained by passage in C57BL/6 mice (H0099/13). In total, 6-8-week old mice were infected by oral gavage with 200 L3 larvae. On indicated days, mice were killed by isofluorane inhalation followed by cervical dislocation.

Adult parasite fecundity was determined by counting eggs shed by female worms kept individually for $24 \mathrm{~h}$ (eight per donor mouse) in 96-well plates in Roswell Park Memorial Institute medium (RPMI) containing $1 \%$ fetal bovine serum (FCS) $200 \mathrm{U} \mathrm{ml}^{-1}$ penicillin and $200 \mu \mathrm{g} \mathrm{ml}^{-1}$ streptomycin (all from PAA, Wien, Austria).

Preparation of single-cell suspensions. Spleens, mLN, and PP were isolated and placed in cold RPMI1640 containing $1 \% \mathrm{FCS}, 100 \mathrm{U} \mathrm{ml}^{-1}$ penicillin and $100 \mu \mathrm{g} \mathrm{ml}^{-1}$ streptomycin (PAA).

PP were digested in $1 \mathrm{ml}$ RPMI1640 containing $0,1 \mathrm{mg} \mathrm{ml}^{-1}$ Liberase (Roche, Basel, Switzerland) and $0,1 \mathrm{mg} \mathrm{m}^{-1}$ DNase (Sigma, St Louis, MO, USA), in addition to $1 \% \mathrm{FCS}, 100 \mathrm{U} \mathrm{ml}^{-1}$ penicillin and $100 \mu \mathrm{g} \mathrm{ml}^{-1}$ streptomycin (PAA) at $37^{\circ} \mathrm{C}$ on a shaker for $30 \mathrm{~min}$. Spleens, $\mathrm{mLN}$, and pre-digested PP were forced through $70 \mu \mathrm{m}$ cell strainers (BD Bioscience, San Jose, CA, USA) to obtain single-cell suspensions.

For isolation of small intestinal lamina propria cells, the entire small intestine was excised and placed in ice cold HBSS (w/o Ca2 ${ }^{+}, \mathrm{Mg}^{+}{ }^{+}$) containing $2 \%$ FCS and $10 \mathrm{~mm}$ HEPES (PAA). Tissues were thoroughly washed in HBSS/FCS/HEPES (Hank's Balanced Salt Solution/FCS/ 4-(2-hydroxyethyl)-1-piperazineethanesulfonic acid) followed by cutting into $1 \mathrm{~cm}$ pieces and placed in $20 \mathrm{ml} \mathrm{HBSS/FCS/}$ HEPES containing $0,154 \mathrm{mg} \mathrm{ml}^{-1}$ DTE (Sigma). Tissues were incubated in a tube shaker water bath $\left(200 \mathrm{rpm}, 37^{\circ} \mathrm{C}\right)$ for $15 \mathrm{~min}$. This was repeated twice before adding intestinal pieces to $20 \mathrm{ml} \mathrm{HBSS/FCS/}$ HEPES containing $5 \mathrm{mM}$ ethylenediaminetetraacetic acid followed by stirring at room temperature for $15 \mathrm{~min}$. This was repeated three more times. Residual ethylenediaminetetraacetic acid was removed by rinsing intestinal pieces in RPMI 1640 before placing them in $10 \mathrm{ml}$ $37^{\circ} \mathrm{C}$ RPMI1640 containing $0,1 \mathrm{mg} \mathrm{ml}^{-1}$ Liberase and $0,1 \mathrm{mg} \mathrm{ml}^{-1}$ DNAse in addition to $10 \%$ FCS. Following incubation, tubes were vortexed vigorously to disrupt remaining tissue pieces and the whole suspension was forced up and down through an $18 \mathrm{G}$ needle, then filtered over a $70 \mu \mathrm{m}$ cell strainer and washed twice with HBSS/HEPES. Cell suspensions were layered on a percoll gradient and lamina propria cells collected from the $40 / 70 \%$ interface.

Antibody detection by enzyme-linked immunosorbent assay. Total antibody titers were detected in sera by sandwich enzyme-linked immunosorbent assay. Intestinal $\operatorname{IgA}$ was measured in supernatants from $2 \mathrm{~cm}$ of duodenal organ cultures (opened longitudinally and cultured for $24 \mathrm{~h}$ at $37^{\circ} \mathrm{C}$ ).

To determine parasite-specific antibody titers, plates were coated with $H$. polygyrus excretory/secretory products at a concentration of $10 \mu \mathrm{g} \mathrm{ml}^{-1}$ and serially diluted sera from H.polygyrus-challenged mice were added.

Cytokine detection enzyme-linked immunosorbent assay. Cytokines were measured in supernatants of stimulated cells using enzymelinked immunosorbent assay detection kits for mouse IL-4, IL-13, IL-5 (ebioscience, San Diego, CA, USA), and IL-10 (BD bioscience).

Intestinal production of IL- $1 \beta$ and IL- 6 was determined in homogenized duodenum and normalized to protein content based on BCA protein detection (Thermo Fisher, Rockford, IL) and TGF- $\beta$ was detected in the supernatants of duodenal organ cultures and normalized to tissue weight.

Flow cytometry. For surface and intracellular staining, the following monoclonal antibodies were used: B220-PE-Cy7 (RA-B2), T-bet-PECy7 (MI24), PD-1-eFluor780 (J43), IL-10-eFluor660 (JES5-16E3), GATA-3-eFluor660 (TWAJ), CD45-eFluor450 (30-F11), IFN- $\gamma$ eFluor450 (XMG1.2), FoxP3-eFluor450 (FJK-16s), IgM-eFluor450 (eB121-15F9); FceRI-APC (MAR-1); c-Kit-fluoroscein isothiocyanate (2B8); MHCII-PE-Cy5 (M5/114.15.2), CD11c-eFluor450 (N418); CD103-PE (2E7); CD8 $\alpha$-Biotin (53-6.7); (all from eBioscience).
IL-4-PE (11B11), Siglec-F-PE (E50-2440); CCR3-Alexa647 (83103); CXCR5-biotin (2G8) (all from BD biosciences). CD4-PerCP (RM4-5); IgG1-APC (RMG1-1); CD11b-APC-Cy7 (M1/70) (all from BioLegend, San Diego, CA, USA). Fluoroscein isothiocyanate-labeled polyclonal goat anti mouse IgA was from Southern Biotech and biotinylated peanut agglutinin was from Biozol (Eching, Germany). Streptavidin labeled in PE, PE-Cy7, and fluoroscein isothiocyanate were from eBioscience. Dead cells were excluded using eFluor 506 fixable viability dye from eBioscience.

For intracellular staining of cytokines and transcription factors, cells were fixed and permeabilized using the fix/perm eBioscience buffer kit. Cells were analyzed using a Canto II flow cytometer (BD Bioscience) and FlowJo software 9.8.5 (Tree star Inc., Ashland, OR, USA)

Eosinophil cell sorting and cytospin. Small intestine lamina propria single-cell suspensions were prepared as described above and pooled from 3-4 mice. Eosinophils (CD45 ${ }^{+}, \mathrm{SSC}^{\text {hi }}$, SiglecF ${ }^{+}, \mathrm{CCR}^{+}$) were sorted with a FACSAria cell sorter (BD Bioscience). In total, $5 \times 10^{4}$ sorted cells were added to cytospin columns and slides were stained in Diff quick (LT-SYS, Berlin, Germany).

Eosinophil transfer. Blood containing 10-30\% eosinophil was taken from IL-5 transgenic mice and enriched for eosinophils via magnetic bead lymphocyte depletion. In brief, erythrocytes were lysed with hypotonic buffer and $\mathrm{T}$ cells and $\mathrm{B}$ cells were depleted using the following reagents from miltenyi: anti-CD3-Biotin; anti-biotin magnetic beads; anti-CD19 magnetic beads. Eosinophil purity was checked by flow cytometry and was typically $80 \%$.

RT-PCR. RNA was isolated from intestinal tissue using the innuPREP RNA kit following manufacturer's instructions (Analytik Jena, Jena,Germany). In total, $2 \mu \mathrm{g}$ of RNA was reverse transcribed to cDNA using a High Capacity RNA to cDNA kit (Applied Biosystems, Foster City, CA). The relative expression of $\beta$-glucuronidase (Gusb), ariginase-1 (Arg-1), resistin-like molecule-alpha (Relm- $\alpha$ ), and Relm- $\beta$ was determined via real-time PCR using $10 \mathrm{ng}$ of cDNA and FastStart Universal SYBR Green Master Mix (Roche). Primer pairs used for gene amplification were as follows: Gusb fwr: GCTCGGGGCAAATTC CTTTC, rev: CTGAGGTAGCACAATGCCCA. Arg-1 fwr: CAGAAG AATGGAAGAGTCAG, rev: CAGATATGCAGGGAGTCACC. Relm- $\alpha$ (Retnla) fwr: TCCCAGTGAATACTGATGAGA, rev: CCAC TCTGGATCTCCCAAGA. Relm- $\beta$ (Retnlb) fwr: GGCTGTGGATC GTGGGATAT, rev: GAGGCCCAGTCCATGACTGA. Activationinduced cytidine deaminase (Aicda) fwr: CCAGGAACCGCTACT CGTTT, rev: GGTCCGTCTCAGGCACTATG. TGF $\beta$ fwr: CTGCTG ACCCCCACTGATAC, rev: AGCCCTGTATTCCGTCTCCT. Efficiencies for each primer pair were determined by generating a standard curve and mRNA expression was normalized to the housekeeping gene Gusb and calculated by Roche Light Cycler 480 software, using naive $\mathrm{BALB} / \mathrm{c} \mathrm{cDNA}$ as a calibrator.

Histology. Formalin-fixed, paraffin-embedded sections $(1-2 \mu \mathrm{m})$ of duodenum were de-waxed and stained with hematoxylin and eosin for overview, with periodic acid Schiff for goblet cell quantification and by Direct red 80 (Sigma) for the detection of eosinophils. Enteritis was scored using hematoxylin and eosin -stained section as described before. ${ }^{35} \mathrm{PAS}^{+}$goblet cells were counted along five villi per section.

Images were acquired using the AxioImager Z1 microscope (Carl Zeiss MicroImaging, Inc., Göttingen, Germany). All evaluations were performed blind.

Statistics. Experiments were performed as indicated and expressed as mean \pm s.e.m. Statistical analysis was performed using GraphPad Prism software. Unless otherwise indicated, level of significance was tested by one-way analysis of variances followed by Holm-Sidak post hoc test for multiple comparison.

SUPPLEMENTARY MATERIAL is linked to the online version of the paper at http://www.nature.com/mi 


\section{ACKNOWLEDGMENTS}

The authors thank Yvonne Weber, Marion Müller, Bettina Sonnenburg, Christiane Palissa and Beate Anders for providing their excellent technical support. We thank David Vöhringer for the provision of IL-5 transgenic NJ.1638 mice. This work was supported by the Deutsche Forschungsgemeinschaft grant GK1121 and HA2542/3-2 (to S.H.). J.S. received funding from the IMPRS-IDI graduate program.

\section{AUTHORS CONTRIBUTION}

JS, SR and SH contributed to conception and design of experiments and writing of final manuscript. Data collection was performed by JS with the exception of histological data, which were collected by AAK. Data analysis was performed by JS and SR. SS and CB provided valuable input in data interpretation and conceptual input. All authors contributed in reviewing the manuscript.

\section{DISCLOSURE}

The authors declare no conflict of interest.

c 2017 Society for Mucosal Immunology

\section{REFERENCES}

1. Spencer, L.A. \& Weller, P.F. Eosinophils and Th2 immunity: contemporary insights. Immunol. Cell. Biol. 88, 250-256 (2010).

2. Klion, A.D. \& Nutman, T.B. The role of eosinophils in host defense against helminth parasites. J. Allergy Clin. Immunol. 113, 30-37 (2004).

3. Shin, E.-H. et al. Protective roles of eosinophils in nippostrongylus brasiliensis infection. Int. Arch. Allergy Immunol. 114, $45-50$ (1997).

4. Watanabe, K. et al. Strongyloides ratti: the role of interleukin- 5 in protection against tissue migrating larvae and intestinal adult worms. J. Helminthol. 77, 355-361 (2003).

5. Knott, M.L. et al. Impaired resistance in early secondary Nippostrongylus brasiliensis infections in mice with defective eosinophilopoeisis. Int. J. Parasitol. 37, 1367-1378 (2007).

6. Sher, A., Coffman, R.L., Hieny, S. \& Cheever, A.W. Ablation of eosinophil and IgE responses with anti-IL-5 or anti-IL-4 antibodies fails to affect immunity against Schistosoma mansoni in the mouse. J. Immunol. 145, 3911-3916 (1990).

7. Fabre, V. et al. Eosinophil deficiency compromises parasite survival in chronic nematode infection. J. Immunol. 182, 1577-1583 (2009).

8. Walsh, E.R. et al. Strain-specific requirement for eosinophils in the recruitment of Tcells to the lung during the development of allergic asthma. J. Exp. Med. 205, 1285-1292 (2008).

9. Simon, D., Wardlaw, A. \& Rothenberg, M.E. Organ-specific eosinophilic disorders of the skin, lung, and gastrointestinal tract. J. Allergy Clin. Immunol. 126, 3-13 (2010).

10. Rothenberg, M.E. \& Hogan, S.P. The eosinophil. Annu. Rev. Immunol. 24, 147-174 (2006).

11. Linch, S.N. et al. Mouse eosinophils possess potent antibacterial properties in vivo. Infect. Immun. 77, 4976-4982 (2009).

12. Phipps, S. etal. Eosinophils contribute to innate antiviral immunity and promote clearance of respiratory syncytial virus. Blood 110, 1578-1586 (2007).

13. Wu, D. et al. Eosinophils sustain adipose alternatively activated macrophages associated with glucose homeostasis. Science 332, 243-247 (2011).

14. Heredia, J.E. et al. Type 2 innate signals stimulate fibro/adipogenic progenitors to facilitate muscle regeneration. Cell 153, 376-388 (2013).

15. Chu, V.T. et al. Eosinophils are required for the maintenance of plasma cells in the bone marrow. Nat. Immunol. 12, 151-159 (2011).

16. Chu, V.T. et al. Eosinophils promote generation and maintenance of immunoglobulin-A-expressing plasma cells and contribute to gut immune homeostasis. Immunity 40, 582-593 (2014).

17. Yu, C. et al. Targeted deletion of a high-affinity GATA-binding site in the GATA-1 promoter leads to selective loss of the eosinophil lineage in vivo. J. Exp. Med. 195, 1387-1395 (2002).

18. Jacobsen, E.A. et al. Differential activation of airway eosinophils induces $\mathrm{IL}-13$ mediated allergic Th2 pulmonary responses in mice. Allergy 70 , 1148-1159 (2015).
19. Jung, Y. et al. IL-1 $\beta$ in eosinophil-mediated small intestinal homeostasis and IgA production. Mucosal. Immunol. 8, 930-942 (2015).

20. Smith, K.A. \& Maizels, R.M. IL-6 controls susceptibility to helminth infection by impeding Th2 responsiveness and altering the Treg phenotype in vivo. Eur. J. Immunol. 44, 150-161 (2014).

21. Zaiss, M.M. et al. IL-1 $\beta$ suppresses innate IL-25 and IL-33 production and maintains helminth chronicity. PLoS Pathog. 9, e1003531 (2013).

22. Everts, B. et al. Migratory CD103 + dendritic cells suppress helminthdriven type 2 immunity through constitutive expression of IL-12. J. Exp. Med. 213, 35-51 (2015).

23. Lee, J.J. et al. Defining a link with asthma in mice congenitally deficient in eosinophils. Science 305, 1773-1776 (2004).

24. King, \& IL, Mohrs, M. IL-4-producing CD4 + Tcells in reactive lymph nodes during helminth infection are T follicular helper cells. J. Exp. Med. 206, 1001-1007 (2009).

25. Reinhardt, R.L., Liang, H.-E. \& Locksley, R.M. Cytokine-secreting follicular T cells shape the antibody repertoire. Nat. Immunol. 10, 385-393 (2009).

26. Haynes, N.M. et al. Role of CXCR5 and CCR7 in follicular Th cell positioning and appearance of a programmed cell death gene-1 high germinal centerassociated subpopulation. J. Immunol. 179, 5099-5108 (2007).

27. Macpherson, A.J., McCoy, K.D., Johansen, F.-E. \& Brandtzaeg, P. The immune geography of IgA induction and function. Mucosal. Immunol. 1, 11-22 (2008).

28. Cerutti, A. The regulation of IgA class switching. Nat. Rev. Immunol. 8, 421-434 (2008).

29. Muramatsu, M. et al. Specific expression of activation-induced cytidine deaminase (AID), a novel member of the RNA-editing deaminase family in germinal center B cells. J. Biol. Chem. 274, 18470-18476 (1999).

30. Chen, H.-H. et al. Eosinophils from murine lamina propria induce differentiation of naive $T$ cells into regulatory $T$ cells via TGF- $\beta 1$ and retinoic acid. PLoS One 10, e0142881 (2015).

31. McCoy, K.D. et al. Polyclonal and specific antibodies mediate protective immunity against enteric helminth infection. Cell Host Microbe 4, 362-373 (2008).

32. Anthony, R.M. et al. Memory $T(H) 2$ cells induce alternatively activated macrophages to mediate protection against nematode parasites. Nat. Med. 12, 955-960 (2006).

33. Esser-von Bieren, J. et al. Antibodies trap tissue migrating helminth larvae and prevent tissue damage by driving IL-4R $\alpha$-independent alternative differentiation of macrophages. PLoS Pathog. 9, e1003771 (2013).

34. Herbert, D.R. et al. Intestinal epithelial cell secretion of RELM-beta protects against gastrointestinal worm infection. J. Exp. Med. 206, 2947-2957 (2009).

35. Padigel, U.M. et al. Eosinophils act as antigen-presenting cells to induce immunity to strongyloides stercoralis in mice. J. Infect. Dis. 196, 1844-1851 (2007).

36. Chu, D.K. et al. Indigenous enteric eosinophils control DCs to initiate a primary Th2 immune response in vivo. J. Exp. Med. 211, 1657-1672 (2014).

37. Wesa, A.K. \& Galy, A. IL-1 beta induces dendritic cells to produce IL-12. Int. Immunol. 13, 1053-1061 (2001).

38. Griseri, T. et al. Granulocyte macrophage colony-stimulating factoractivated eosinophils Promote Interleukin-23 Driven Chronic Colitis. Immunity 43, 187-199 (2015).

39. Rosenberg, H.F., Masterson, J.C. \& Furuta, G.T. Eosinophils, probiotics, and the microbiome. J. Leukoc. Biol., e-pub before print (doi:10.1189/ jlb.3RI0416-202R)2016).

40. Ma, C.S., Deenick, E.K., Batten, M. \& Tangye, S.G. The origins, function, and regulation of T follicular helper cells. J. Exp. Med. 209, 1241-1253 (2012).

41. Avery, D.T., Bryant, V.L., Ma, C.S., de Waal Malefyt, R. \& Tangye, S.G. IL-21-induced isotype switching to IgG and IgA by Human Naive $\mathrm{B}$ cells is differentially regulated by IL-4. J. Immunol. 181, 1767-1779 (2008).

42. Bansemir, A.D. \& Sukhdeo, M.V. The food resource of adult Heligmosomoides polygyrus in the small intestine. J. Parasitol. 80, 24-28 (1994). 